\title{
Montmorillonite Clay-Based Polyurethane Nanocomposite As Local Triamcinolone Acetonide Delivery System
}

\author{
Flávia Carmo Horta Pinto, ${ }^{1}$ Armando Silva-Cunha, ${ }^{2}$ Gerson Antônio Pianetti, ${ }^{2}$ Eliane \\ Ayres, ${ }^{3}$ Rodrigo Lambert Oréfice, ${ }^{4}$ and Gisele Rodrigues Da Silva ${ }^{1}$ \\ ${ }^{1}$ School of Pharmacy, Federal University of São João Del Rei, MG 35.501-296, Divinópolis, Brazil \\ ${ }^{2}$ School of Pharmacy, Federal University of Minas Gerais, Belo Horizonte, MG, Brazil \\ ${ }^{3}$ Department of Materials, Technologies and Processes, Minas Gerais State University, Belo Horizonte, MG, Brazil \\ ${ }^{4}$ Department of Metallurgical and Materials Engineering, Federal University of Minas Gerais, Belo Horizonte, MG, Brazil
}

Correspondence should be addressed to Gisele Rodrigues Da Silva, giselersilva@ufsj.edu.br

Received 17 May 2011; Revised 6 July 2011; Accepted 12 July 2011

Academic Editor: Donglu Shi

Copyright ( $) 2011$ Flávia Carmo Horta Pinto et al. This is an open access article distributed under the Creative Commons Attribution License, which permits unrestricted use, distribution, and reproduction in any medium, provided the original work is properly cited.

\begin{abstract}
Biodegradable polyurethane was synthesized by preparing aqueous polyurethane dispersion having poly(caprolactone) and poly(ethylene glycol) as soft segments. Montmorillonite particles were delaminated within the waterborne polyurethane to produce a nanocomposite. The triamcinolone acetonide (TA), an important corticoid drug, was dispersed into the nanocomposite followed by a drying step to produce an implantable drug delivery system. Infrared (FTIR) results demonstrated that the original chemical structure of the TA was preserved after incorporation into the nanocomposite. Wide angle (WAXS) and small angle X-ray scattering (SAXS) results suggested that TA and clay do not dramatically change the morphology phase of the polymer although they can interact with each other. The presence of montmorillonite particles in the nanocomposite reduced the rate of TA release as compared to the pure polyurethane and enhanced the mechanical properties of the polymer. The overall results indicate that montmorillonite clay-based polyurethane nanocomposite could be potentially applied as local TA delivery system.
\end{abstract}

\section{Introduction}

Nanoparticles derived from expandable clays, such as montmorillonite, have been considered for more than 15 years as a reinforcing agent for polymers [1]. These clays have an interlayer space than can be promptly impregnated by many species including polymers [2]. Exchanging natural cations present in clay by $\mathrm{Na}^{+}$can allow water to enhance interlayer spacing up to several hundred angstroms [3]. Nonpolar cations can also be used to improve the compatibility between hydrophobic polymers and clays, but these special cations may lead to toxicity when biomedical applications are considered.

Nanocomposites composed on polyurethane and montmorillonite particles have been widely investigated [4-6]. They are usually prepared by dispersing the layered silicate particles into the polyurethane matrix. The resulting polymer-layered silicate nanocomposites frequently display advanced properties (such as mechanical properties) due to their high surface area $[1,7]$. Many properties, such as mechanical [8], water vapor permeability [5], and water permeability $[9,10]$ can be improved by incorporating small amounts of delaminated clay to a polyurethane matrix [11]. Montmorillonite clay-based polyurethane nanocomposites have been also explored as implantable drug-delivery systems. In this case, nanocomposites were loaded with therapeutic agents and then implanted in specific organs. They would be able to control the release of a therapeutic agent to a specific target to treat or prevent different types of pathological processes [12-14]. All these studies demonstrated the potential applicability of nanocomposites composed on polyurethane and montmorillonite in different fields.

Intercalated and/or exfoliated morphologies can be obtained when clay nanoparticles are included into polymers [2]. An intercalated nanocomposite is obtained when polymer chains impregnate the clay interlayer gap to produce a 
multilayered structure. The properties of this type of composite are usually similar to polymers containing micrometer particles. In the exfoliated or delaminated structure, clay layers are well dispersed within the polymer matrices, and polymer-clay interactions are dramatically enhanced [15, 16]. Exfoliation of clays within polymer is usually associated with large improvements in properties not always possible to be obtained with micrometer particles are used [17].

In the preparation of polyurethane/clay nanocomposites, modifications of montmorillonite by using organic modifiers such as alkylammonium to improve the compatibility between hydrophilic organomontmorillonite and hydrophobic polyurethanes are often employed $[3,6,18,19]$. These organic chains having positively charged ends can be bonded to the surface of the negatively charged silicate layers resulting in an increase of the interlayer gap [20,21]. However, these modifications can be potentially toxic when considering biomedical applications of the yielded devices. To avoid this type of potential toxicity, montmorillonite without any modification can be intercalated and/or delaminated using aqueous polyurethane dispersions to produce nanocomposites. In this type of nanocomposite processing technique, water is used to enlarge the space between silicate layers $[10,22,23]$.

In this study, polyurethane based on poly(caprolactone) and poly(ethylene glycol), as soft segment, and isophorone diisocyanate and hydrazine, as hard segments, was synthesized and dispersed in water. Montmorillonite particles and triamcinolone acetonide were dispersed directly in the waterborne polyurethane. The triamcinolone acetonide-loaded polyurethane nanocomposite implants were obtained by cutting the nanocomposite films into the desired shape (squares of $5 \mathrm{~mm}$ of side). The structure of the implantable devices was characterized by analytical techniques to identify not only the intercalation and/or exfoliation of the montmorillonite onto the nanocomposite, but also how the drug interacted and affected its morphology. The effect of the clay particles distribution on the mechanical properties of the polyurethane was also evaluated. The analyzed structure of the implants was useful in understanding the in vitro drug-controlled release. Additionally, the in vivo release profile of the drug was evaluated through their subcutaneous implantation on the back of mice. Finally, it was revealed the applicability of the montmorillonite clay-based polyurethane nanocomposite loaded with triamcinolone acetonide as local controlled drug-delivery systems.

\section{Materials and Methods}

2.1. Synthesis of the Aqueous Polyurethane Dispersion (PU). Aqueous polyurethane dispersion (PU) was prepared by a prepolymer mixing process, using a $250 \mathrm{~mL}$ three-neck glass flask equipped with a heating mantel, a mechanical stirrer, and a thermometer. The macrodiol components-polycaprolactone-diol (PCL 1000) (Tone Polyol 2221, $\left.\bar{M}_{n}=1000 \mathrm{~g} \cdot \mathrm{mol}^{-1}\right)$, polycaprolactone-diol (PCL 2000) (Tone Polyol 0249, $\bar{M}_{n}=2000 \mathrm{~g} \cdot \mathrm{mol}^{-1}$ ) (DowUSA) (Sigma-Aldrich), and poly(ethylene glycol) (PEG,
TABle 1: Composition (wt.\%) of the aqueous PU dispersion*.

\begin{tabular}{lc}
\hline Reagents & Concentration (wt.\%) \\
\hline Isophorone diisocyanate (IPDI) & 8.58 \\
Polycaprolactone-diol 1000 (PCL1000) & 4.85 \\
Polycaprolactone-diol 2000 (PCL2000) & 9.09 \\
Polyethylene glycol 1500 (PEG 1500) & 0.73 \\
2,2-bis(hydroxymethyl) propionic acid & 0.97 \\
(DMPA) & 0.73 \\
Triethylamine (TEA) & 74.70 \\
Water & 1.08 \\
Hydrazine (HZ)
\end{tabular}

${ }^{*} 0.01 \%$ of dibutyl tin dilaurate based on the amounts of IPDI, PCL, and DMPA.

$M_{n}=1500 \mathrm{~g} \cdot \mathrm{mol}^{-1}$, Sigma-Aldrich), isophorone diisocyanate (IPDI) (Bayer-Brazil) (NCO/OH ratio of 2.3) and 2,2bis(hydroxymethyl) propionic acid (DMPA) (98.3\%, Fluka) were added to the reactor in the presence of dibutyl tin dilaurate (DBDLT) (Miracema Nuodex-Brazil) and the reaction was carried out at $70^{\circ} \mathrm{C}-75^{\circ} \mathrm{C}$ in a nitrogen atmosphere for $4 \mathrm{~h}$. The amount of free $\mathrm{NCO}$ groups on a percentage basis was determined by the standard dibutyl amine back titration method. After titration, the prepolymer temperature was allowed to drop to $40^{\circ} \mathrm{C}$. The carboxylic acid groups were neutralized by the addition of triethylamine (TEA) (98\%, Vetec-Brazil). The mixture was stirred for another $40 \mathrm{~min}$ to ensure that the reaction had been completed. All samples were dispersed by adding deionized water to the neutralized prepolymer which was stirred vigorously. After the dispersion, the amount of hydrazine (HZ) (solution 64\%, Arch Química-Brazil) enough to react with free NCO groups was added to the reactor with a small amount of water, and stirring was continued for another $30 \mathrm{~min}$. This chemical procedure was successful in producing a polyurethane dispersion with a solid content of about $25 \%$. The composition of the polyurethane dispersion is shown in Table 1 . Films were produced by casting the dispersion in a Teflon mould and allowing them to dry at room temperature for one week. Afterwards the films were placed in an oven at $60^{\circ} \mathrm{C}$ for $24 \mathrm{~h}$ [24]. A schematic representation of the chemical reactions and processing steps used to produce the polyurethane dispersion and nanocomposites is shown in Figure 1.

2.2. Incorporation of Clay Nanoparticles-Cloisite $\mathrm{Na}^{+}$. The montmorillonite clay without any treatment (Cloisite $\mathrm{Na}^{+}$, Southern Clay Products) was dispersed in water ( $5 \mathrm{wt} . \%)$ by stirring it for 24 hours at room temperature. This procedure is useful to reduce the average particle size of the clay particles to yield clay nanoparticles dispersed in water [25]. Clay nanoparticles were incorporated into the polyurethane aqueous dispersion by mixing the polymer with the aqueous dispersion of the clay (Figure 1). The dispersions were mixed in proportions to yield a final nanocomposite containing 5 wt. $\%$ of clay in the polyurethane. 


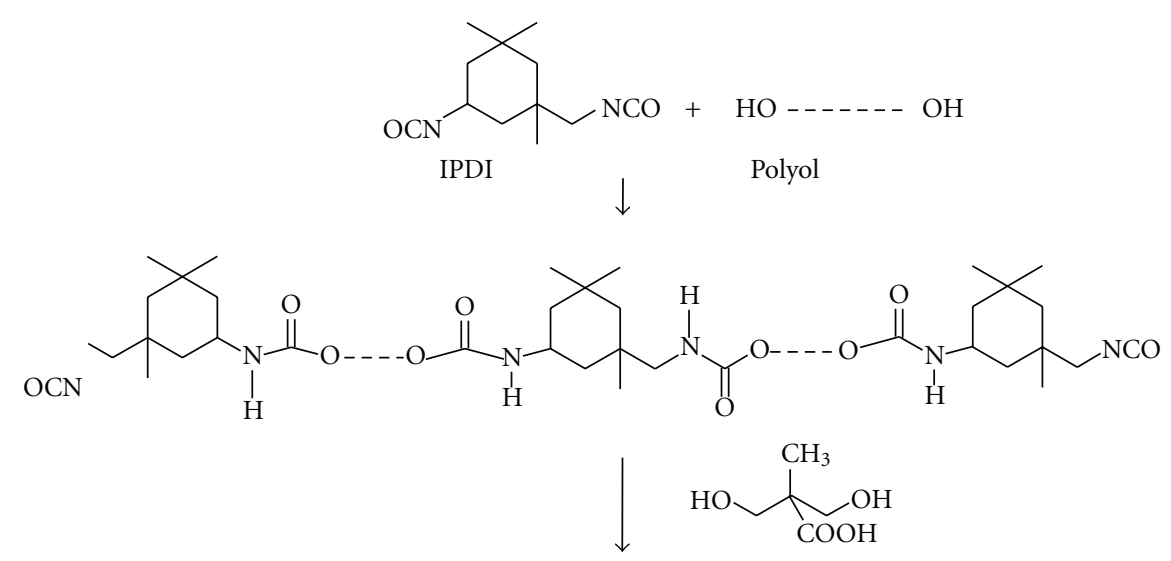

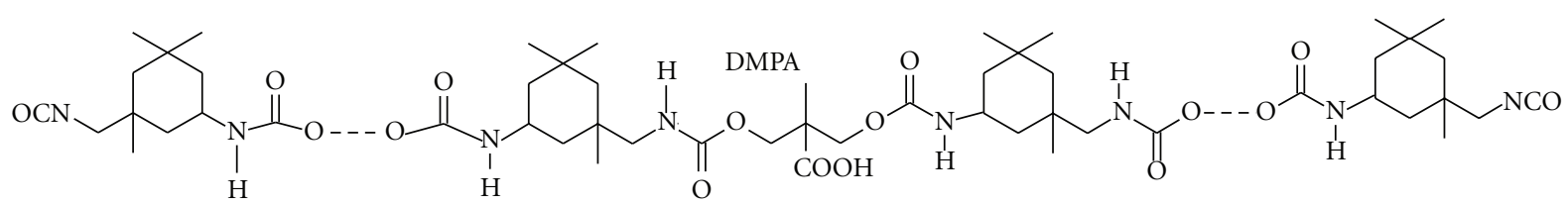

NCO terminated prepolymer

$$
\downarrow \begin{gathered}
\mathrm{N}(\mathrm{Et})_{3} \\
\text { Triethyl amine }
\end{gathered}
$$

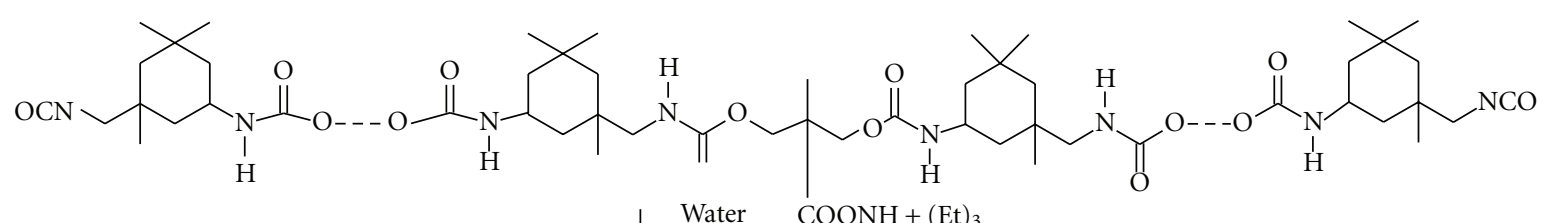

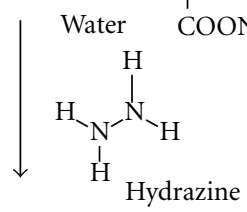

Polyurethane aqueous dispersion

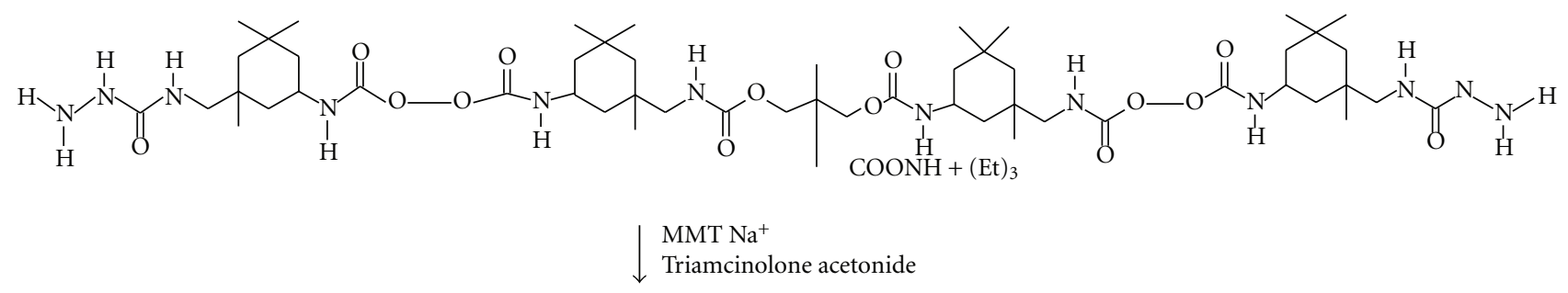

PUD containing MMT and drug

FIGURE 1: Schematic representation of the process used to prepare polyurethane containing montmorillonite $\left(\mathrm{MMT}-\mathrm{Na}^{+}\right)$and triamcinolone acetonide.

2.3. Incorporation of Triamcinolone Acetonide and Preparation of the Implants. Triamcinolone acetonide (TA) (Sigma-Aldrich, $99.0 \%$ ) was incorporated into the polymer by dispersing it in the nanocomposite prior of casting the films to yield materials having $20.5 \mathrm{wt} \%$ of the drug (Figure 1). The dried films were cut into squares ( $5 \mathrm{~mm}$ in side) to obtain the triamcinolone acetonide-loaded polyurethane nanocomposite implants (TA PU-NC implants) having approximately $1.27 \mathrm{mg}$ each one. Triamcinolone acetonide-loaded polyurethane implants (without clay particles) (TA PU implants) were also prepared.

\subsection{Characterization}

2.4.1. Fourier Transform Infrared. Infrared spectra were collected in a Fourier transform infrared spectrophotometer (FTIR; Perkin Elmer, model Spectrum 1000). Measurements 
were carried out using the attenuated total reflectance (ATR) technique. Each spectrum was a result of 32 scans with a resolution of $4 \mathrm{~cm}^{-1}$.

2.4.2. Small Angle X-Ray Scattering. The measurements of synchrotron small angle X-ray scattering (SAXS) were performed using the beam line of the national synchrotron light laboratory (LNLS, Campinas, Brazil). The photon beam used in the LNLS SAXS beamline comes from one of the 12 bending magnets of the electron storage ring. The white photon beam is extracted from the ring through a high-vacuum path. After passing through a thin beryllium window, the beam is monochromatized $(\lambda=1.608 \AA)$ and horizontally focused by a cylindrically bent and asymmetrically cut silicon single crystal. The focus is located at the detection plane. The Xray scattering intensity, $I(q)$, was experimentally deter-mined as a function of the scattering vector " $q$ " whose modulus is given by $q=(4 \pi / \lambda) \sin (\theta)$, where $\lambda$ is the X-ray wavelength and $\theta$ being half the scattering angle. Each SAXS pattern corresponds to a data collection time of 900 s. From the experimental scattering intensity produced by all the studied samples, the parasitic scattering intensity produced by the collimating slits was subtracted. All SAXS patterns were corrected for the nonconstant sensitivity of the PSD, for the time varying intensity of the direct synchrotron beam and for differences in sample thickness. Because of the normalization procedure, the SAXS intensity was determined for all samples in the same arbitrary units so that they can be directly compared.

2.4.3. Wide Angle X-Ray Scattering. Wide angle X-ray scattering (WAXS) was performed by using a synchrotron light beam with a wavelength of $1.608 \AA$ and an exposition time of $300 \mathrm{~s}$. The scattering intensity was recorded by a Pilatus $(100 \mathrm{~K}, 33 \mathrm{~mm} \times 84 \mathrm{~mm})$ detector. The sample to detector distance used was $80 \mathrm{~mm}$.

2.4.4. Scanning Electron Microscopy. Scanning electron microscopy (SEM) was performed using a JEOL microscope (model JSM-6360LV) operating at $15 \mathrm{kV}$. The TA PU implants were cryofractured and mounted on aluminium stubs using double-sided adhesive tape. Prior to microscopic examination, all the samples were sputter-coated with a gold layer under argon atmosphere using a sputter apparatus (Balzers Union SCD 040 unit, Balzers, Germany). The implants surfaces were viewed at 1000-10000x magnification, and the images were transferred to the computer by means of a digital image transference interface (DITI). The photomicrographs were adjusted using the software Adobe Photoshop 6.0 and Adobe Illustrator 9.01 (Adobe Systems Incorporated, 2000, USA).

2.4.5. Mechanical Tests. Mechanical tensile tests were performed by following information reported in the ASTM D638. Samples were cut from sheets using a dog-bone die and tested using a $1.0 \mathrm{~mm} / \mathrm{min}$ load rate and a load cell of $200 \mathrm{~N}$.
2.5. In Vitro Release of TA from the PU-NC and PU Implants. The United States Pharmacopeia [26] states in the general chapter $\langle 1092\rangle$ the dissolution procedure: "sink conditions are defined as the volume of medium at least three times that required to form a saturated solution of drug substance. When sink conditions are present, it is more likely that dissolution results will reflect the properties of the dosage form".

The in vitro release of TA was carried out under sink conditions during 8 months. As the aqueous solubility of TA is $21.0 \mu \mathrm{g} / \mathrm{mL}$ at $28^{\circ} \mathrm{C}$ [27], sink conditions were achieved with at least $181 \mathrm{~mL}$ for the evaluated polymeric implants.

The TA PU-NC and TA PU implants were placed in different Erlenmeyers containing $181 \mathrm{~mL}$ of phosphate buffer solution ( $\mathrm{PBS} \mathrm{pH}=7.4)(n=5)$. These Erlenmeyers were placed inside an incubator set at $37^{\circ} \mathrm{C}$ and $30 \mathrm{rpm}$. At predetermined intervals, $181 \mathrm{~mL}$ of the PBS was sampled and the same volume of fresh PBS was added to each tube. The amount of TA released from each PU-NC and PU implants was assayed by high performance liquid chromatographic method described above, and expressed as the cumulative percentage of TA released in the medium. The average of the obtained measurements was calculated and used to plot the release profile curve.

The TA released from PU-NC and PU implants was measured by high-performance liquid chromatography (HPLC) by a Hewlett Packard HP 1100 apparatus equipped with an autosampler model HP 1100. An HP 1100 pump was set at a constant flow rate of $1.2 \mathrm{~mL} / \mathrm{min}$. A C-18 reversed-phase column $(4.6 \mathrm{~mm} \times 250 \mathrm{~mm}$, macropore size of $5 \mu \mathrm{m})$ (Chromolith RP-18E, Merck KGaA Performance \& Life Science Chemicals, Germany) was also used. The mobile phase was a mixture of acetonitrile (EM Science, Merck KGaA, Germany) and ultrafiltrated water obtained from Milli Q Plus, Millipore (USA) (50:50). An HP 1100 ultraviolet detector was used at a wavelength of $242 \mathrm{~nm}$. The HPLC method was previously validated, and demonstrated to be specific, linear, precise and accurate as well as simple and reliable.

2.6. In Vivo Release of TA from the PU-NC and PU Implants. Six-to-eight-week-old female Swiss mice from the Centro de Bioterismo of the Federal University of Minas Gerais (UFMG) were maintained in individual cages, with food and water ad libitum, and controlled temperature and humidity in the animal house of the School of Pharmacy of the Federal University of São João Del Rei (UFSJ). Experiments were approved by the Ethics Committee in Animal Experimentation at UFSJ.

The animals were anesthetized with a mixture of $\left(10 \mathrm{mg} \mathrm{kg}^{-1}\right)$ xylazine and $\left(100 \mathrm{mg} \mathrm{kg}^{-1}\right)$ ketamine hydrochloride (i.p.). Their dorsal hair was shaved and the skin wiped with $70 \%$ ethanol. TA PU-NC and TA PU implants were incorporated into the sponge discs, and they were aseptically inserted into a subcutaneous pouch that had been made with curved artery forceps through a $1 \mathrm{~cm}$ long dorsal mid-line incision. After the implantation procedure, the animals were maintained in individual cages and provided with chow pellets and water ad libitum. The light/dark cycle was $12: 12 \mathrm{~h}$ with lights on at 7:00 am and lights off at 7:00 pm. 
Postoperatively, the animals were monitored for any signs of infection at the operative site, or upon discomfort or distress; any mice presenting such signs were immediately sacrificed.

TA PU-NC and TA PU implants were inserted into a subcutaneous pouch of the mice as described above. At 4, 7, 15, and 30 days after implantation, animals ( $n=5$ for each time) were euthanized, and the TA PU-NC and TA PU implants were carefully removed. The implant was fragmented using a scissor, and the pieces were dissolved in $50 \mathrm{~mL}$ of a mixture of acetonitrile and ultrafiltrated water (1:1). The content of TA remaining in the PU-NC and PU implants was measured by HPLC method previously described.

\section{Results and Discussion}

The incorporation of clay nanoparticles into the polymer matrix affected the mechanical properties of the polymer as initially predicted. Clay nanoparticles improved the elastic modulus of the polymer approximately two and a half times when compared to the pure polymer, as indicated in Table 2. Therefore, clay particles could be used to tune the stiffness of the PU, avoiding the necessity of changing some of the parameters of the PU synthesis, such as the ratio between soft and hard segments. The adjustment of the stiffness of the nanocomposite is an attractive characteristic for designing biomedical scaffolds and implantable solid systems [13], since stiff needle-shaped implants can be easily inserted through soft tissues without the need of a special incision. The reduction in both the strength and strain failure rates is a consequence of the interactions between the clay and polymer chains that restrict chain mobility.

Figure 2 shows the infrared spectra of pure PU (Figure 2(a)), PU-NC (containing clay) (Figure 2(b)), TA PU implants (Figure 2(c)), TA PU-NC implants (Figure 2(d)), pure TA (Figure 2(e)), and pure montmorillonite (Figure 2(f)). Characteristic infrared bands observed in pure PU include $2945-2866 \mathrm{~cm}^{-1}$ related to the stretching vibration of $-\mathrm{CH}_{2}$ groups and $1723-1600 \mathrm{~cm}^{-1}$ equivalent to the stretching vibration of free and hydrogen-bonded carbonyl in the urethane, urea, and ester bonds in the poly(caprolactone) segments. The hydrogen bonded carbonyl in the urethane and urea (in the hard segment) and ester bonds in poly(caprolactone) (in the soft segment) could interact not only with the polar surface of the clay nanoparticles to yield a higher degree of hydrogen bonded species, but also with the primary amine present in the molecular structure of the polyurethane. The absorption band that resulted from stretching vibrations of bonded primary amine was observed in $3333 \mathrm{~cm}^{-1}$, confirming the existence of interaction between the hydrogen of the primary amine and carbonyl in the urethane, urea, and ester groups. However, an absorption infrared band at $1531 \mathrm{~cm}^{-1}$ was also identified corresponding to bending vibrations of nonbonded primary amine. An absorption band around $2270 \mathrm{~cm}^{-1}$ was not observed in spectra, which indicated the absence of nonreactive NCO groups [28]. If this type of chemical group was present, the occurrence of side reactions which leads to the formation of allophanate
TABLE 2: Mechanical properties of PU and PU-NC films.

\begin{tabular}{lccc}
\hline & $\begin{array}{c}\text { Elastic modulus } \\
(\mathrm{MPa})\end{array}$ & Strength $(\mathrm{MPa})$ & $\begin{array}{c}\text { Strain at failure } \\
(\%)\end{array}$ \\
\hline PU & 125 & 33 & 944 \\
PU-NC & 333 & 25 & 503 \\
\hline
\end{tabular}

or biuret linkages would be possible [29]. The same infrared bands observed in pure PU were identified in spectra of PU-NC (Figure 2(b)), TA PU implants (Figure 2(c)) and TA PU-NC implants (Figure 2(d)).

The infrared spectra of pure clay (Figure 2(f)) revealed a weak infrared band at $3600 \mathrm{~cm}^{-1}$ due to the $-\mathrm{OH}$ band stretch in $\mathrm{Al}-\mathrm{OH}$ and $\mathrm{Si}-\mathrm{OH}$, and an intense absorption band at approximately $1040 \mathrm{~cm}^{-1}$ due to Si-O stretching (out-ofplane) and $\mathrm{Si}-\mathrm{O}$ stretching (in-plane) vibration in layered silicates $[30,31]$. The infrared band corresponding to Si-O bonds is also observed in the spectra of PU-NC and TA PU-NC implants (Figures 2(b) and 2(d)), but the intensity is lower than that present in the spectra of pure clay. It is suggested that the intensity change in the $\mathrm{Si}-\mathrm{O}$ stretching vibration band is related to the low concentration of clay in polyurethane, the weakening of interactions between interlayer water molecules and surface oxygen [32], and also due to the intercalation of the polyurethane and/or TA between clay lamellas.

Typical absorption bands observed in pure TA (Figure 2(e)) can be visualized in the spectra of TA PU (Figure 2(c)) and TA PU-NC implants (Figure 2(d)), such as $3392 \mathrm{~cm}^{-1}$ corresponding to the hydroxyl groups, between $3000-2900 \mathrm{~cm}^{-1}$ related to $\mathrm{C}-\mathrm{H}$ stretching of $\mathrm{sp} 3$ and $\mathrm{sp} 2$ carbons, $1700-1730 \mathrm{~cm}^{-1}$ and $1662 \mathrm{~cm}^{-1}$ due to carbonyl groups of aliphatic ester and ketone. The obtained FTIR results corroborated with those previously reported [33]. The maintenance of the characteristic infrared bands of the PU, PU-NC, and TA, as well as the absence of new bands, demonstrated that there was no detectable chemical interaction between the polymer and drug during the manufacture process of the implants and indicated that the drug was successfully incorporated into the polyurethanenanocomposite.

Figure 3 shows wide-angle $\mathrm{x}$-ray scattering (WAXS) of pure PU (Figure 3(a)), PU-NC (containing clay) (Figure 3(b)), TA PU implants (Figure 3(c)), TA PU-NC implants (Figure 3(d)), and pure TA (Figure 3(e)). The WAXS curve of the pure PU (Figure 3(a)) revealed that no defined scattering peaks can be noted as a consequence of the presence of a highly amorphous structure. Crystallization of poly(caprolactone) segments is restricted, having low molar mass $(<2000 \mathrm{~g} / \mathrm{mol})$ in polyurethanes, since low molar mass oligomers are confined between hard domains that do not allow them to have regular conformations that can lead to crystallization [34]. The WAXS curve recorded for PU containing clay nanoparticles (Figure 3(b)) revealed also an amorphous pattern of the nanocomposite. According to Kiersnowski et al. [35], the WAXS curve of montmorillonite reveals diffraction peaks related to the crystalline structure of this clay. By contrast, WAXS data obtained for PU-NC 


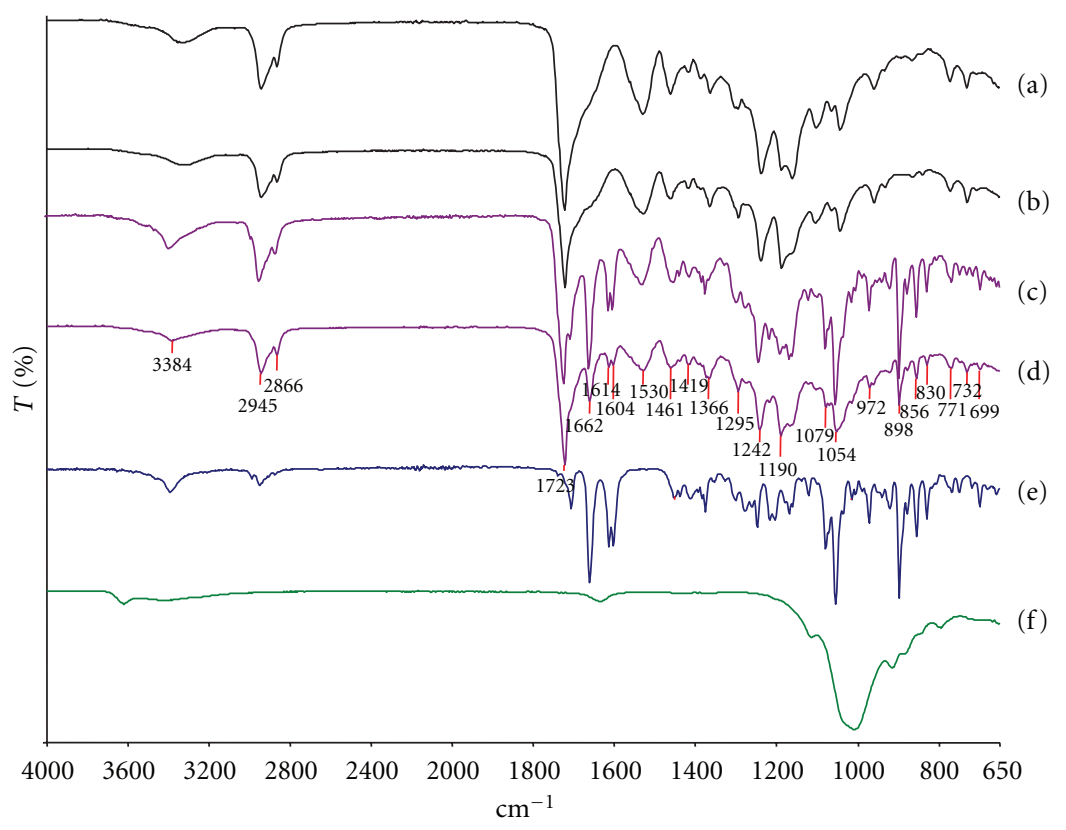

FIGURE 2: FTIR spectra of pure PU (a), PU-NC (containing clay) (b), TA PU implants (c), TA PU-NC implants (d), pure TA, (e) and pure montmorillonite (f).

did not show diffraction peaks, suggesting the disruption of the regular layered structure of the montmorillonite are due to the delamination of the clay incorporated into the polymer. The presence of poly(ethylene glycol) segments within the polyurethane macromolecular architecture was useful in promoting complete exfoliation of the clay structure, since the hydrophilic poly(ethylene glycol) possibly interacted efficiently with the polar clay surface to restrict clay nanoparticles to pack again during drying of the aqueous dispersion [36]. Typical scattering peaks observed in pure TA (Figure 3(e)) can be visualized in the WAXS curves of TA PU (Figure 3(c)) and TA PU-NC implants (Figure 3(d)), demonstrating the preserved crystalline pattern of the drug. However, the incorporation of clay nanoparticles and drug into the polyurethane led to a small shift of the diffraction peaks of TA to lower angles. This shift in $q$ values suggested interactions between drug and clay nanoparticles due to the diffusion of the TA into the clay sheets and crystallization within the clay lamella, leading to the enlargement of the distance between crystalline planes of the drug. The interactions between silicate layers and guest molecules, such as nonionic TA, are weak interaction bonds represented by hydrogen bonding and hydrophobic interactions (Van der Walls). Complexes with montmorillonite and anionic or other nonionic drugs also exhibited bonding via absorption due to the establishment of weak interaction bonds [37]. On the other hand, McGinity and Lach [38] confirmed that basic molecules bonded strongly with montmorillonite, since clay minerals are naturally inorganic cationic exchangers and they may undergo ion exchange with basic drugs.

Small angle X-ray scattering (SAXS) has been used to characterize the morphology of nanoscaled phase-separated

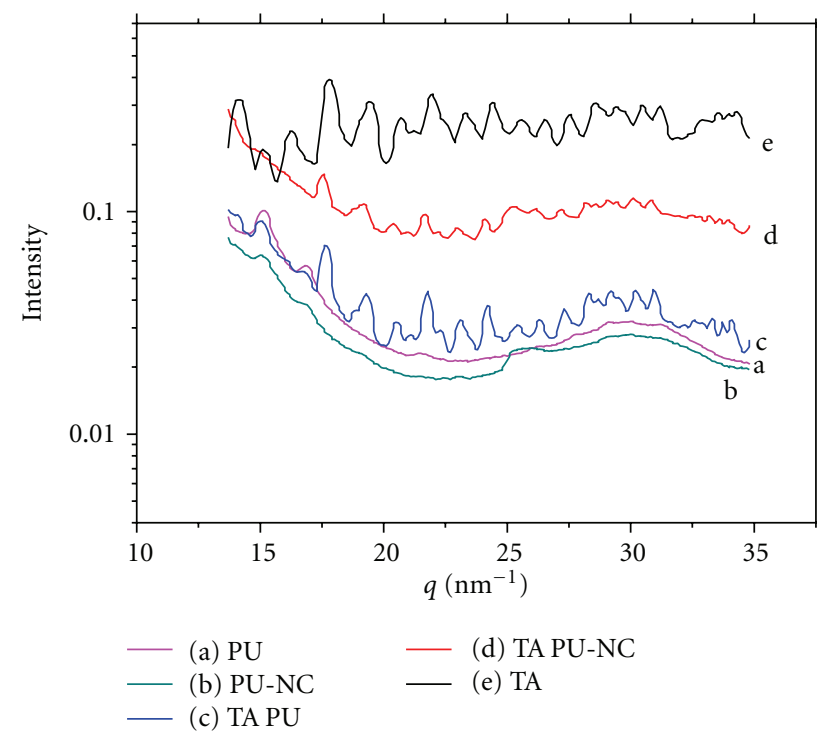

Figure 3: WAXS curves of pure PU (a), PU-NC (containing clay) (b), TA PU implants (c), TA PU-NC implants (d), and pure TA (e).

materials, such as polymer nanocomposites, block copolymers, and segmented polymers, since this technique records electron density fluctuations throughout the material in a nanometer range $[39,40]$. SAXS provides useful data to study segmental polymers such as polyurethanes, since this type of polymer often phase separate in hard and soft domains that have enough electronic contrast to affect X-ray scattering at low angles. Nanocomposites containing clay has also 
been investigated by SAXS $[41,42]$ to show the degree of clay delamination and nanoparticle orientation within the polymer.

Figure 4 shows SAXS data for PU-based materials that comprise nanoparticles derived from clay and TA. SAXS data in Figure 4 was reformatted using the Lorentz correction $\left(I_{(q)} q^{2}\right)$, that is, useful to enhance the resolution of small SAXS peaks. No scattering peak can be clearly seen in SAXS curve associated with the pure PU (Figure 4(a)) even after the Lorentz correction, indicating the low electron difference between segments of the polyurethane. Considering the fact that this polyurethane is based on poly(caprolactone) and poly(ethylene glycol), as soft segments, and urethane hard segments, the hydrophilic domains were able to chemically interact by means of hydrogen bonds, producing a more homogeneous structure with possibly very small domains with no major differences in electron density. The incorporation of TA into the polymer did not lead to major changes in the scattering profile (Figure 4(c)). Therefore, the presence of TA did not alter the original structure of pure PU. Otherwise, an increase in scattering (mainly for lower values of q) was observed when clay nanoparticles were introduced into PU (Figure 4(b)) as an evidence of the presence of these nanoentities that have a higher electron density than the pure polymer. It was demonstrated previously [43] that clay nanoparticles can interact with hard segments of polyurethane through the formation of hydrogen bonds to change the course of the phase separation process. Additionally, the polar clay surface can also interact with the poly(ethylene glycol)-based soft segments of the polymer, changing the morphology of the polyurethane. The level of scattering was even more enhanced when TA was also incorporated into PU together with clay nanoparticles (Figure 4(d)). This result may indicate that interactions between the drug and clay may have created a larger number of scattering sites within the polymer. The interactions between silicate layers and TA, accommodated in their gallery spaces, might be hydrophobic interactions (Van der Walls) and hydrogen bonding, since the drug was absorbed in the clay mainly in the neutral form [37].

Scanning electron microscopy (SEM) was used for examination of the fractured surfaces of pure PU (Figure 5(a)), PU-NC (containing clay) (Figure 5(b)), TA PU implants (Figure 5(c)), and TA PU-NC implants (Figure 5(d)). The cross-sectional area of the pure PU was smooth and dense, with no evidence of pores or channels. On the other hand, the PU-NC surface was completely rough due to the homogeneous distribution of the clay nanoparticles in the polymer surface. This could be attributed to the chemical interactions between the polar clay surface and polar urethane bonds and/or poly(ethylene glycol) present in the segments of the polyurethane. The cross-sectional area of the TA PU-NC implants was also rough due to the presence of the clay nanoparticles and TA crystals not only in the PU matrix, but also on the clay sheets. Since no large clay and TA aggregates could be observed on the fractured surface of the samples, it can be suggested that the manufacture procedure of the implants

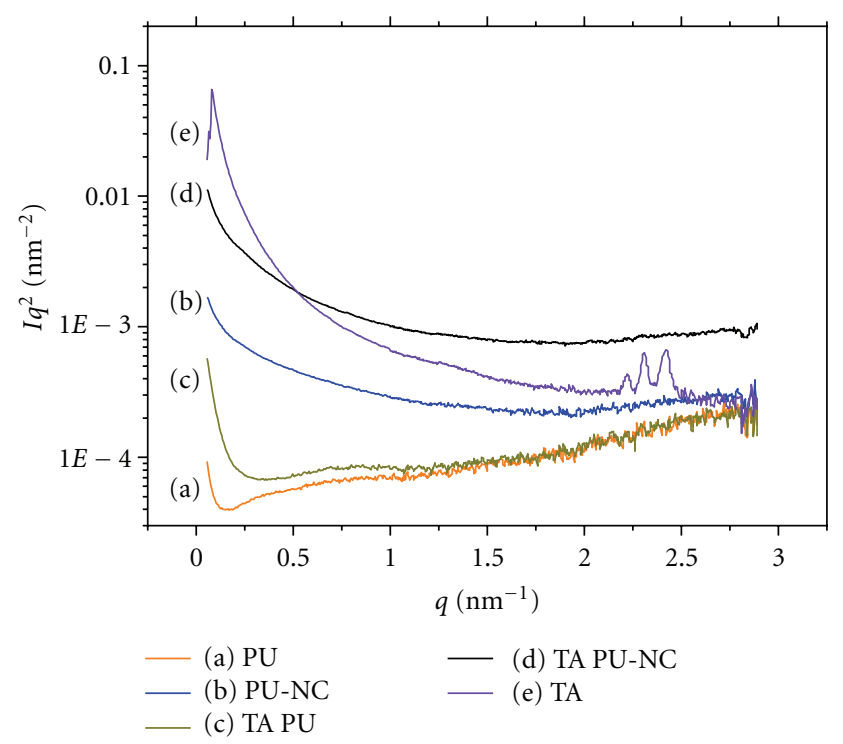

FIGURE 4: Lorentz corrected SAXS scattering data of pure PU (a), PU-NC (containing clay) (b), TA PU implants (c), TA PU-NC implants (d), and pure TA (e).

yielded a high-quality polyurethane nanocomposite loaded with small crystals of the drug.

Figure 6 shows the percentage of the cumulative TA release profile from PU implants (Figure 6(a)) and PU-NC implants (Figure 6(b)). The TA leached from the implantable devices was quantified by HPLC method. Neither any peak for a degradation product nor any alteration of the chromatographic pattern of TA was observed, indicating that the preparation technique of the PU and PU-NC implants and the conditions of the in vitro release of the drug did not affect the stability of the drug. TA PU and TA PU-NC implants led to a controlled release of the drug which followed a bimodal pattern: during the first 15 days, a faster dissolution and diffusion of the drug was released corresponding to a minimal burst effect. Over this period, approximately $16 \%$ and $24 \%$ of TA was released from PU$\mathrm{NC}$ and PU implants, respectively, corresponding to the leaching of TA crystals deposited in the implant surfaces. In the second stage, approximately $93 \%$ and $85 \%$ of $\mathrm{TA}$ was released from the PU and PU-NC implants. It suggests that this phase was characterized by the diffusion of the drug and the degradation of the polymeric matrix. The presence of poly(ethylene glycol) in the soft segments of the polyurethane increased hydrophilicity and aqueous permeability in the polymer backbone, promoting hydrolytic cleavage of ester bonds of the poly(caprolactone) also present in the soft segments of the polyurethane. Furthermore, the scission of the poly(caprolactone) increased the porosity and surface area for drug diffusion [44]. In terms of the bonds present in poly(ester urethanes), the most susceptible to hydrolysis are ester bonds. However, the hydrolysis of ester bonds of urethane groups in one order of magnitude is slower relative to ester bonds of the soft segment. Therefore, the soft segment bonds hydrolyze at a greater rate than 


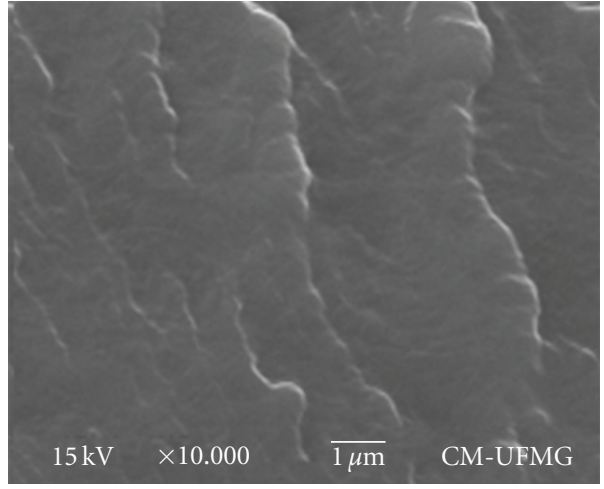

(a)

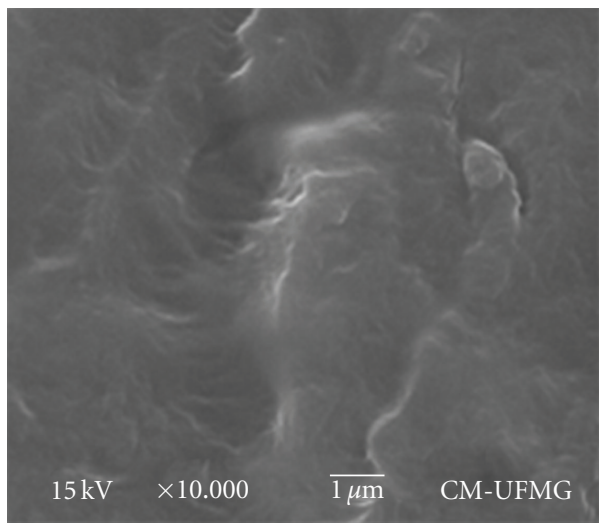

(c)

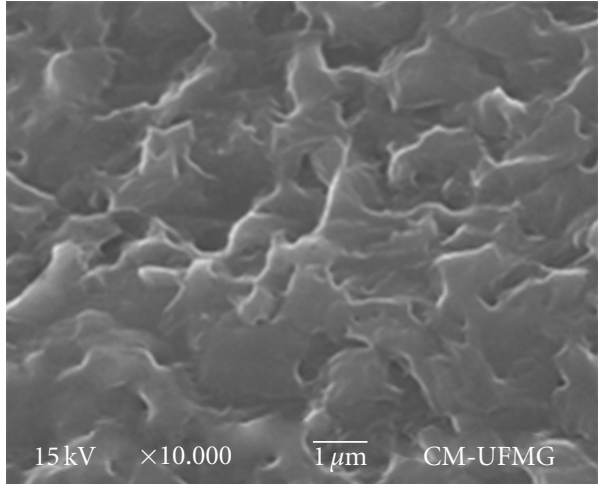

(b)

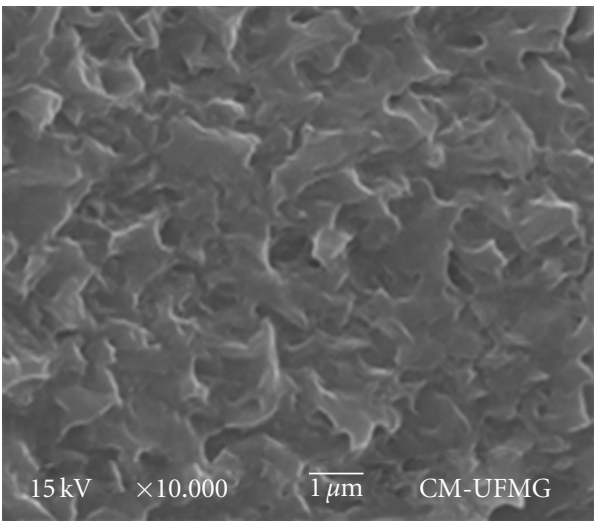

(d)

FIGURE 5: SEM images of pure PU (a), PU-NC (containing clay) (b), TA PU implants (c), and TA PU-NC implants (d).

hard segment bonds and govern the degradation rate of the poly(ester urethanes) [45].

The incorporation of montmorillonite particles into the polymeric matrix loaded with the drug reduced the rate of TA release over the time. This may be due to the establishment of different types of host-guest interactions between clay nanoparticles and TA intercalated into montmorillonite (as detected by WAXS and SAXS). Besides the interactions between them being weak interaction bonds, they contributed to a more extensive release of the drug from the PU-NC implants.

Figure 7 shows the percentage of the cumulative TA release profile from PU implants (Figure $7(\mathrm{a})$ ) and PUNC implants (Figure 7(b)). The TA-loaded PU and PUNC implants maintained a sustained release of the drug for at least 30 days within the subcutaneous tissue of the mice. The PU and PU-NC implants showed an initial burst release of the drug over the first 7 days. Over this period, approximately $36 \%$ and $27 \%$ of TA was released from PU and PU-NC implants, indicating faster dissolution of the drug deposited on the surface of the polymeric implants. In a second stage that occurred between the 8th and the 30th days of the test, the PU and PU-NC implants released $80 \%$ and $73 \%$ of TA, respectively, providing controlled and sustained TA release within the subcutaneous tissue of the mice. During this period, the dissolution and diffusion of

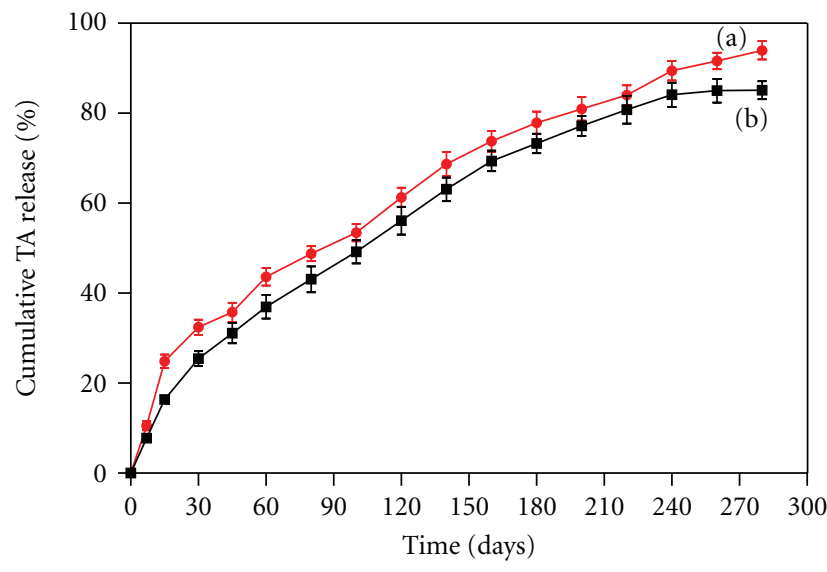

Figure 6: Cumulative TA release profile from PU implants (a) and PU-NC implants (b). Results represent mean \pm standard deviation ( $n=5$ for each time and each sample).

the drug and the degradation of the polymeric matrix might modulate the TA release rate. It was previously described that the in vivo degradation of polyurethanes involves the hydrolysis of ester linkages in polyester soft segments, and the enzymatic hydrolysis by the presence of esterases, since those enzymes are able to recognize and act upon unnatural 


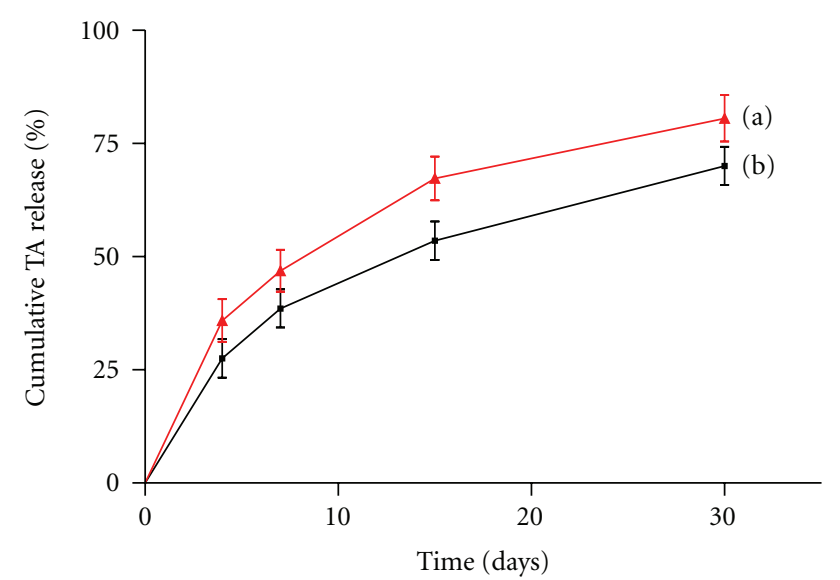

FIgURE 7: Cumulative TA release profile from PU (a) and PU-NC (b) subcutaneous implants in mice. Sustained release of TA was observed up to day 30 after implantation. Results represent mean \pm standard deviation ( $n=5$ for each time and each sample).

substrates such as polyurethanes [46]. Additionally, the overall rate of TA released from the PU-NC implants was lower than that obtained for the PU implants. Therefore, the intercalation capability offered by the montmorillonite particles was applied to decrease the release of the TA, providing a prolonged delivery of the drug over the time in comparison with the free polymer. This can be relevant for long-term treatment of chronic inflammatory pathologies.

It was observed that approximately $31 \%$ of TA was released from PU-NC implants in vitro and $73 \%$ of drug was delivered by PU-NC implants in vivo during 30 days. The differences between the physiological environment (in vivo test) and the simulated physiological conditions (in vitro test) explain the lack of correlation between the in vivo and in vitro profile release of TA from PU-NC implants.

\section{Conclusion}

In the present work, the montmorillonite without any treatment could be intercalated within the polymer chains due to the use of aqueous polyurethane dispersion to produce the nanocomposite. The triamcinolone acetonide, an important corticoid, was incorporated into the nanocomposite by dispersing it in the waterborne polyurethane. Triamcinolone acetonide-loaded polyurethane nanocomposite implants were successfully designed. The incorporation of nanocomposite into the polymer chains modulated the mechanical properties of the polymer system that became more attractive not only for designing implantable solid systems but also for biomedical scaffolds. FTIR and WAXS data showed that the incorporation of the drug into the nanocomposite did not severely change the chemical and crystallographic aspects of the drug respectively. SAXS data demonstrated that the introduction of the clay nanoparticles into the polyurethane provided no major modifications of the morphology of the polymer. However, this technique provided some evidences that clay can interact with the drug. In vitro and in vivo triamcinolone acetonide release studies showed that the drug was released slower from the nanocomposite compared to the pure polyurethane, as a result of the interaction between the montmorillonite and the drug present in the interlayer spacing of the host clay. Therefore, the incorporation of montmorillonite nanoparticles into the biodegradable polyurethane chains was useful not only to tune the mechanical properties of the polymer but also to provide controlled long-term delivery of the drug over the time. The treatment of chronic inflammatory diseases by montmorillonite clay-based polyurethane implants seems feasible.

\section{Acknowledgments}

The authors would like to acknowledge the financial support received from the following institutions: FAPEMIG (Minas Gerais-Brazil), CNPq/MCT (Brazil), and CAPES/MEC (Brazil).

\section{References}

[1] E. P. Giannelis, "Polymer layered silicate nanocomposites," Advanced Materials, vol. 8, no. 1, pp. 29-35, 1996.

[2] A. K. Barick and D. K. Tripathy, "Effect of organically modified layered silicate nanoclay on the dynamic viscoelastic properties of thermoplastic polyurethane nanocomposites," Applied Clay Science, vol. 52, no. 3, pp. 312-321, 2011.

[3] E. Ayres, R. L. Oréfice, and D. Sousa, "Influence of bentonite type in waterborne polyurethane nanocomposite mechanical properties," Macromolecular Symposia, vol. 245-246, pp. 330336, 2006.

[4] A. Cheng, S. Wu, D. Jiang, F. Wu, and J. Shen, "Study of elastomeric polyurethane nanocomposites prepared from grafted organic-montmorillonite," Colloid and Polymer Science, vol. 284, no. 9, pp. 1057-1061, 2006.

[5] M. M. Rahman, H. D. Kim, and W. K. Lee, "Preparation and characterization of waterborne polyurethane/clay nanocomposite: effect on water vapor permeability," Journal of Applied Polymer Science, vol. 110, no. 6, pp. 3697-3705, 2008.

[6] N. Salahuddin, A. Abo-El-Enein, A. Selim, and O. Salah ElDien, "Synthesis and characterization of polyurethane/organo-montmorillonite nanocomposites," Applied Clay Science, vol. 47, no. 3-4, pp. 242-248, 2010.

[7] M. Alexandre and P. Dubois, "Polymer-layered silicate nanocomposites: preparation, properties and uses of a new class of materials," Materials Science and Engineering, vol. 28, no. 1, pp. 1-63, 2000.

[8] Y. I. Tien and K. H. Wei, "High-tensile-property layered silicates/polyurethane nanocomposites by using reactive silicates as pseudo chain extenders," Macromolecules, vol. 34, no. 26, pp. 9045-9052, 2001.

[9] B. Han, A. Cheng, G. Ji, S. S. Wu, and J. Shen, "Effect of organophilic montmorillonite on polyurethane/montmorillonite nanocomposites," Journal of Applied Polymer Science, vol. 91, no. 4, pp. 2536-2542, 2004.

[10] G. R. Da Silva, A. Da Silva Cunha, E. Ayres, and R. L. Oréfice, "Effect of the macromolecular architecture of biodegradable polyurethanes on the controlled delivery of ocular drugs," Journal of Materials Science: Materials in Medicine, vol. 20, no. 2, pp. 481-487, 2009. 
[11] S. Pavlidou and C. D. Papaspyrides, "A review on polymerlayered silicate nanocomposites," Progress in Polymer Science, vol. 33, no. 12, pp. 1119-1198, 2008.

[12] G. R. Da Silva, E. Ayres, R. L. Orefice, S. A. L. Moura, D. C. Cara, and A. Da Silva Cunha, "Controlled release of dexamethasone acetate from biodegradable and biocompatible polyurethane and polyurethane nanocomposite," Journal of Drug Targeting, vol. 17, no. 5, pp. 374-383, 2009.

[13] G. R. Da Silva, A. Da Silva-Cunha, F. Behar-Cohen, E. Ayres, and R. L. Oréfice, "Biodegradable polyurethane nanocomposites containing dexamethasone for ocular route," Materials Science and Engineering C, vol. 31, no. 2, pp. 414-422, 2011.

[14] M. Smith, Y. Omidi, and M. Gumbleton, "Primary porcine brain microvascular endothelial cells: biochemical and functional characterisation as a model for drug transport and targeting," Journal of Drug Targeting, vol. 15, no. 4, pp. 253$268,2007$.

[15] P. Kiliaris and C. D. Papaspyrides, "Polymer/layered silicate (clay) nanocomposites: an overview of flame retardancy," Progress in Polymer Science, vol. 35, no. 7, pp. 902-958, 2010.

[16] J. K. Pandey, K. Raghunatha Reddy, A. Pratheep Kumar, and R. P. Singh, "An overview on the degradability of polymer nanocomposites," Polymer Degradation and Stability, vol. 88, no. 2, pp. 234-250, 2005.

[17] P. C. Lebaron, Z. Wang, and T. J. Pinnavaia, "Polymer-layered silicate nanocomposites: an overview," Applied Clay Science, vol. 15, no. 1-2, pp. 11-29, 1999.

[18] M. Zanetti, P. Bracco, and L. Costa, "Thermal degradation behaviour of PE/clay nanocomposites," Polymer Degradation and Stability, vol. 85, no. 1, pp. 657-665, 2004.

[19] A. Pegoretti, A. Dorigato, M. Brugnara, and A. Penati, "Contact angle measurements as a tool to investigate the filler-matrix interactions in polyurethane-clay nanocomposites from blocked prepolymer," European Polymer Journal, vol. 44, no. 6, pp. 1662-1672, 2008.

[20] A. S. Zerda and A. J. Lesser, "Intercalated clay nanocomposites: morphology, mechanics, and fracture behavior," Journal of Polymer Science Part B: Polymer Physics, vol. 39, no. 11, pp. 1137-1146, 2001.

[21] G. M. Kim, D. H. Lee, B. Hoffmann, J. Kressler, and G. Stöppelmann, "Influence of nanofillers on the deformation process in layered silicate/polyamide-12 nanocomposites," Polymer, vol. 42, no. 3, pp. 1095-1100, 2001.

[22] J. M. Yeh, C. T. Yao, C. F. Hsieh et al., "Preparation, characterization and electrochemical corrosion studies on environmentally friendly waterborne polyurethane/Na+-MMT clay nanocomposite coatings," European Polymer Journal, vol. 44, no. 10, pp. 3046-3056, 2008.

[23] R. C. M. Dias, A. M. Góes, R. Serakides, E. Ayres, and R. L. Oréfce, "Porous biodegradable polyurethane nanocomposites: preparation, characterization, and biocompatibility tests," Materials Research, vol. 13, no. 2, pp. 211-218, 2010.

[24] E. Ayres, R. L. Oréfice, and M. I. Yoshida, "Phase morphology of hydrolysable polyurethanes derived from aqueous dispersions," European Polymer Journal, vol. 43, no. 8, pp. 35103521, 2007.

[25] G. Lagaly and S. Ziesmer, "Colloid chemistry of clay minerals: the coagulation of montmorillonite dispersions," Advances in Colloid and Interface Science, vol. 100-102, pp. 105-128, 2003.

[26] United States Pharmacopeial Convention, The United States Pharmacopeia, United States Pharmacopeial Convention Inc., Rockville, Md, USA, 31th edition, 2008.
[27] L. H. Block and R. N. Patel, "Solubility and dissolution of triamcinolone acetonide," Journal of Pharmaceutical Sciences, vol. 62, no. 4, pp. 617-621, 1973.

[28] I. H. L. Pereira, E. Ayres, P. S. Patrício et al., "Photopolymerizable and injectable polyurethanes for biomedical applications: synthesis and biocompatibility," Acta Biomaterialia, vol. 6, no. 8, pp. 3056-3066, 2010.

[29] V. Thomas and M. Jayabalan, "A new generation of high flex life polyurethane urea for polymer heart valve-studies on in vivo biocompatibility and biodurability," Journal of Biomedical Materials Research-Part A, vol. 89, no. 1, pp. 192-205, 2009.

[30] H. T. Lee and L. H. Lin, "Waterborne polyurethane/clay nanocomposites: novel effects of the clay and its interlayer ions on the morphology and physical and electrical properties," Macromolecules, vol. 39, no. 18, pp. 6133-6141, 2006.

[31] G. V. Joshi, B. D. Kevadiya, H. A. Patel, H. C. Bajaj, and R. V. Jasra, "Montmorillonite as a drug delivery system: intercalation and in vitro release of timolol maleate," International Journal of Pharmaceutics, vol. 374, no. 1-2, pp. 53-57, 2009.

[32] C. M. L. Preston, G. Amarasinghe, J. L. Hopewell, R. A. Shanks, and Z. Mathys, "Evaluation of polar ethylene copolymers as fire retardant nanocomposite matrices," Polymer Degradation and Stability, vol. 84, no. 3, pp. 533-544, 2004.

[33] A. A. da Silva-Junior, J. R. de Matos, T. P. Formariz et al., "Thermal behavior and stability of biodegradable spray-dried microparticles containing triamcinolone," International Journal of Pharmaceutics, vol. 368, no. 1-2, pp. 45-55, 2009.

[34] P. Ping, W. Wang, X. Chen, and X. Jing, "Poly( $\varepsilon$-caprolactone) polyurethane and its shape-memory property," Biomacromolecules, vol. 6, no. 2, pp. 587-592, 2005.

[35] A. Kiersnowski, M. Serwadczak, E. Kułaga et al., "Delamination of montmorillonite in serum-A new approach to obtaining clay-based biofunctional hybrid materials," Applied Clay Science, vol. 44, no. 3-4, pp. 225-229, 2009.

[36] G. R. da Silva, A. da Silva-Cunha, F. Behar-Cohen, E. Ayres, and R. L. Oréfice, "Biodegradation of polyurethanes and nanocomposites to non-cytotoxic degradation products," Polymer Degradation and Stability, vol. 95, no. 4, pp. 491-499, 2010.

[37] C. Aguzzi, P. Cerezo, C. Viseras, and C. Caramella, "Use of clays as drug delivery systems: possibilities and limitations," Applied Clay Science, vol. 36, no. 1-3, pp. 22-36, 2007.

[38] J. W. McGinity and J. L. Lach, "In vitro adsorption of various pharmaceuticals to montmorillonite," Journal of Pharmaceutical Sciences, vol. 65, no. 6, pp. 896-902, 1976.

[39] R. L. Oréfice, E. Ayres, M. M. Pereira, and H. S. Mansur, "Using the nanostructure of segmented polyurethanes as a template in the fabrication of nanocomposites," Macromolecules, vol. 38, no. 10, pp. 4058-4060, 2005.

[40] B. Chu and B. S. Hsiao, "Small-angle X-ray scattering of polymers," Chemical Reviews, vol. 101, no. 6, pp. 1727-1761, 2001.

[41] A. Bafna, G. Beaucage, F. Mirabella, and S. Mehta, "3D hierarchical orientation in polymer-clay nanocomposite films," Polymer, vol. 44, no. 4, pp. 1103-1115, 2003.

[42] M. Song, H. S. Xia, K. J. Yao, and D. J. Hourston, "A study on phase morphology and surface properties of polyurethane/ organoclay nanocomposite," European Polymer Journal, vol. 41, no. 2, pp. 259-266, 2005.

[43] I. M. Pereira, S. Carvalho, M. M. Pereira, M. F. Leite, and R. L. Oréfice, "Effect of the degree of clay delamination on the phase morphology, surface chemical aspects, and properties of hydrolyzable polyurethanes for periodontal regeneration," 
Journal of Applied Polymer Science, vol. 114, no. 1, pp. 254-263, 2009.

[44] B. Li, K. V. Brown, J. C. Wenke, and S. A. Guelcher, "Sustained release of vancomycin from polyurethane scaffolds inhibits infection of bone wounds in a rat femoral segmental defect model," Journal of Controlled Release, vol. 145, no. 3, pp. 221230, 2010.

[45] L. Tatai, T. G. Moore, R. Adhikari et al., "Thermoplastic biodegradable polyurethanes: the effect of chain extender structure on properties and in-vitro degradation," Biomaterials, vol. 28, no. 36, pp. 5407-5417, 2007.

[46] J. P. Santerre, K. Woodhouse, G. Laroche, and R. S. Labow, "Understanding the biodegradation of polyurethanes: from classical implants to tissue engineering materials," Biomaterials, vol. 26, no. 35, pp. 7457-7470, 2005. 

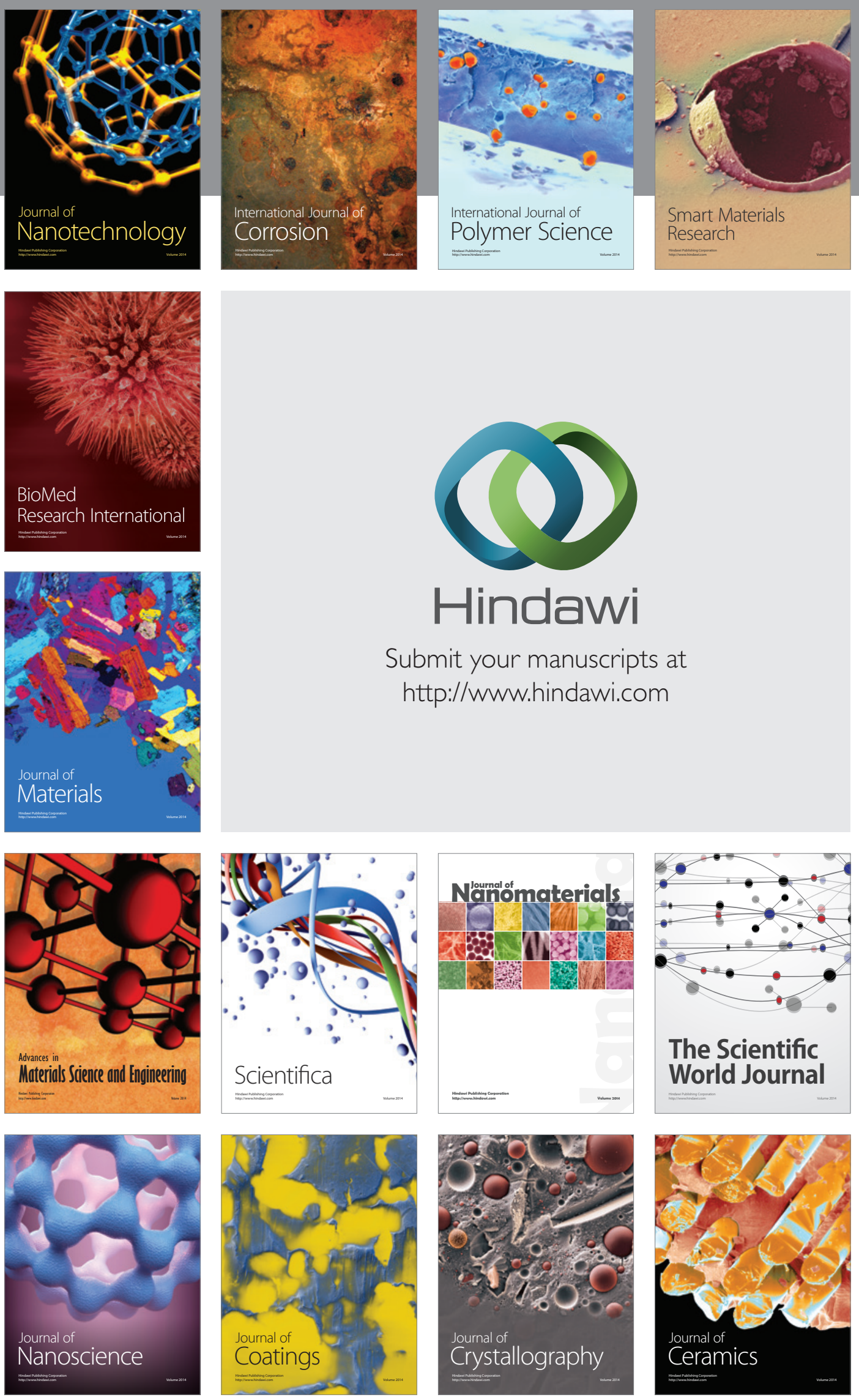

The Scientific World Journal

Submit your manuscripts at

http://www.hindawi.com

\section{World Journal}

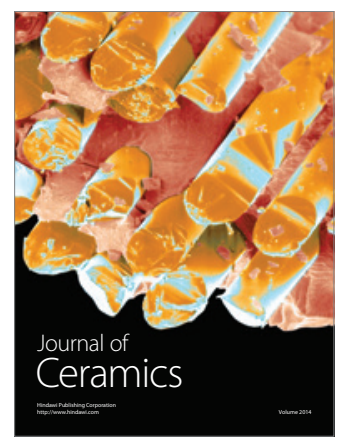

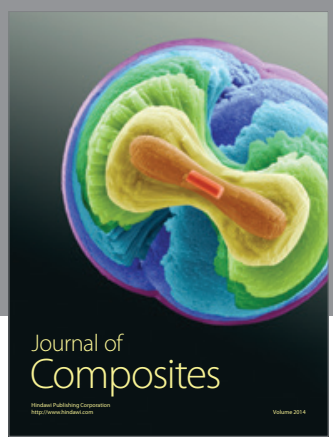
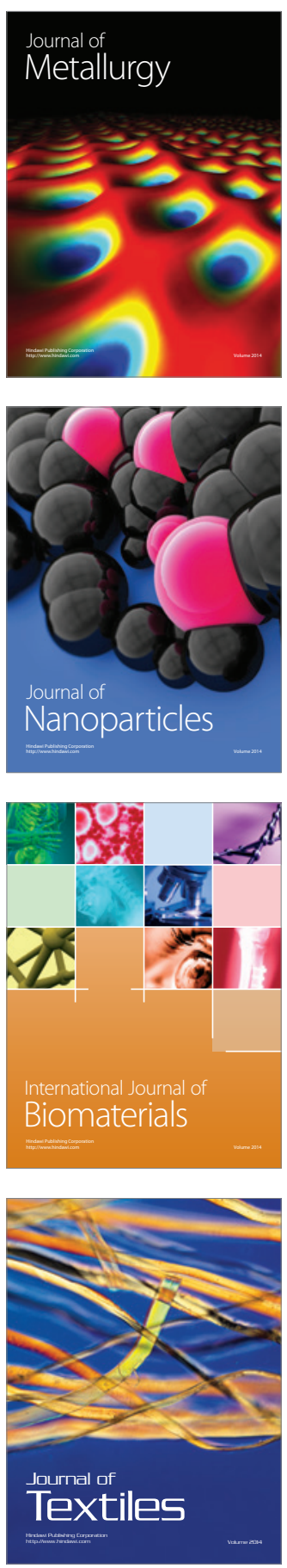